Www.jmscr.igmpublication.org

Impact Factor 5.244

Index Copernicus Value: 83.27

ISSN (e)-2347-176x ISSN (p) 2455-0450

crossref DOI: _https://dx.doi.org/10.18535/jmscr/v4i12.19

\title{
Clinical Profile of Acute Kidney Injury: A Comparative Study of Rifle Vs Akin Criteria
}

\author{
Authors \\ Dr Saurabh Sharma, Dr Vedavathi $R$ \\ KIMS \& RC \\ Email:Vedavathi.r19@gmail.com
}

\begin{abstract}
Acute kidney injury (AKI) is a worldwide clinical dilemma encountered in critically ill patients and characteristically portends an increase in morbidity and mortality.

Objective: To study prospectively the clinical spectrum of AKI and assessment of RIFLE Criteria vs AKIN Criteria in terms of clinical course, risk stratification and prognosis among patients with AKI in ICUs and emergency.

Methodology: Prospective clinical descriptive study was done in 100 patients admitted in various ICUs (Intensive Care Unit) and Emergency Ward in Kempegowda institute of Medical Science. According to patients co morbidities and underlying pathology patients were followed clinically and by serial biochemical tests and were started on conservative treatment, if indicated patients were taken for hemodialysis.

Results: In this study out of 100 patients 48 required ventilator support and inotropic support and 30 patients underwent dialysis. Overall 49 patients died amounting to $49 \%$ mortality. On RIFLE staging mortality in patients with AKI stage failure was $56.2 \%$, injury was $44.4 \%$, and risk is $53.8 \%$ indicating that there was no significant difference in mortality by RIFLE stage.

Conclusion: The AKIN criteria improve the sensitivity of the Acute Kidney Injury (AKI) diagnosis over the RIFLE Criteria. The AKIN Criteria do not improve on the ability of the RIFLE criteria in predicting in hospital mortality of critically ill Patients.
\end{abstract}

\section{Introduction}

Acute kidney injury (AKI) is characterised by sudden Impairment of kidney function resulting in retention of nitrogenous and other waste products.. Acute kidney Injury is a serious complication in the context of severe disease, especially when associated with sepsis and multiple organ dysfunction syndrome. Acute kidney injury has replaced the term acute renal failure $^{1}$. It is manifested with changes in urine output, creatinine and blood urea nitrogen ${ }^{2}$. AKI is common worldwide and is linked with significant morbidity, mortality, and increases the risk for the development of chronic kidney disease $(\mathrm{CKD})^{3-6}$. Utilizing the Kidney Disease: Improving Global Outcome (KDIGO) definition, a meta-analysis demonstrated that the incidence of AKI in adults and children were $21.6 \%$ and $33.7 \%$, individually, the AKI-associated mortality rates were $23.9 \%$ and $13.8 \%$ in adults and children, respectively ${ }^{7}$. AKI is a typical and essential diagnostic and therapeutic dispute for clinicians ${ }^{8}$. More than 200 distinct meaning of AKI were provided ${ }^{9}$. These various definitions make clinical disarray and 
trouble in diagnosing this condition ${ }^{10}$. Several classifications for AKI have been made during the past few years to better define this disease. A consensus definition of AKI was circulated by the Acute Dialysis Quality Initiative (ADQI) in 2004. This consensus definition is termed the Risk/ Injury/Failure/Loss/End-stage (RIFLE) criteria, and the following categories were used: 'Risk' is the least rigorous category of AKI, followed by 'Injury', 'Failure', 'Loss' and 'End-stage renal disease'. In 2007, a customized version of the RIFLE criteria were published by the AKI Network (AKIN) - known as the AKIN criteria ${ }^{11}$. Definition of AKI: the categories of Risk, Injury, and Failure. Since then, many studies compared the two measures to evaluate the incidence and risk factor of AKI, some studies evaluated the sensitivity and accuracy of the RIFLE and AKIN criteria for critically ill patients, some authors. The sensitivity and accuracy of the Risk/ Injury/Failure/Loss/End-stage (RIFLE) versus acute kidney injury Network (AKIN) criteria for acute kidney injury (AKI) in patients remains uncertain

Many studies have compared RIFLE with AKIN in Acute kidney disease. The objective is to study prospectively the clinical spectrum of AKI and assessment of RIFLE Criteria vs AKIN Criteria in terms of clinical course, risk stratification and prognosis among patients with AKI in ICUs and emergency

\section{Methodology}

Prospective clinical descriptive study was done between November 2011 to October 2013. 100 patients admitted in various ICUs (Intensive Care Unit)and Emergency Ward in Kempegowda institute of Medical Science, Bangalore in Department of Medicine were enrolled in the study. All Patients admitted to ICU/Emergency ward under the Department of Medicine were included in the study.Patients with acute and chronic Kidney Disease, Hypertension $>5$ years, Diabetes Mellitus >5 Years, Congestive Cardiac failure were excluded from the study.
A detailed history and physical examination were done as per Performa. Blood Routine-Hb,TC, DC, ESR, Urine Routine, Urine Culture and sensitivity, Blood Culture \& sensitivity, Blood Urea, Serum Creatinine, Serum Electrolytes, Hourly Urine output monitoring and daily I/O Chart, Random Blood Sugar, FBS, PPBS, LFT, ECG, Chest X-RAY, USG-Abdomen and Pelvis, eGFR to be calculated using MDRD formula. Specific Investigations: According to patients co morbidities and underlying pathology patients were followed clinically and by serial biochemical tests and were started on conservative treatment, if indicated patients were taken for hemodialysis.

\section{Results}

In this study, patient between the age group 18-80 years were analysed and the mean age was $40.22 \pm 14.2$ years and for males it was $38.4 \pm 12.48$ years and for females it was $43.5 \pm 16.6$ years.

\section{Treatment and Mortality}

In this study out of 100 patients 48 required ventilator support and inotropic support and 30 patients underwent dialysis. Overall 49 patients died amounting to $49 \%$ mortality.

In this study sepsis accounted for 33 patients, acute gastroenteritis for 20 patients, snake bite for 11 patients, leptospirosis for 9 patients, dengue for 8 patients, hepatorenal syndrome for 6 patients, aluminiumphospide poisoning for 3 patients and paraquat poisoning for 2 patients of AKI. There were 3 patients of unknown poisoning who presented with AKI. There was one patient each of Cresol Poisoning, dengue and Malaria coinfection, Malaria, OP Compound poisoning. (Fig 1) 
Figure 1. Causes of Acute Kidney Injury(AKI)

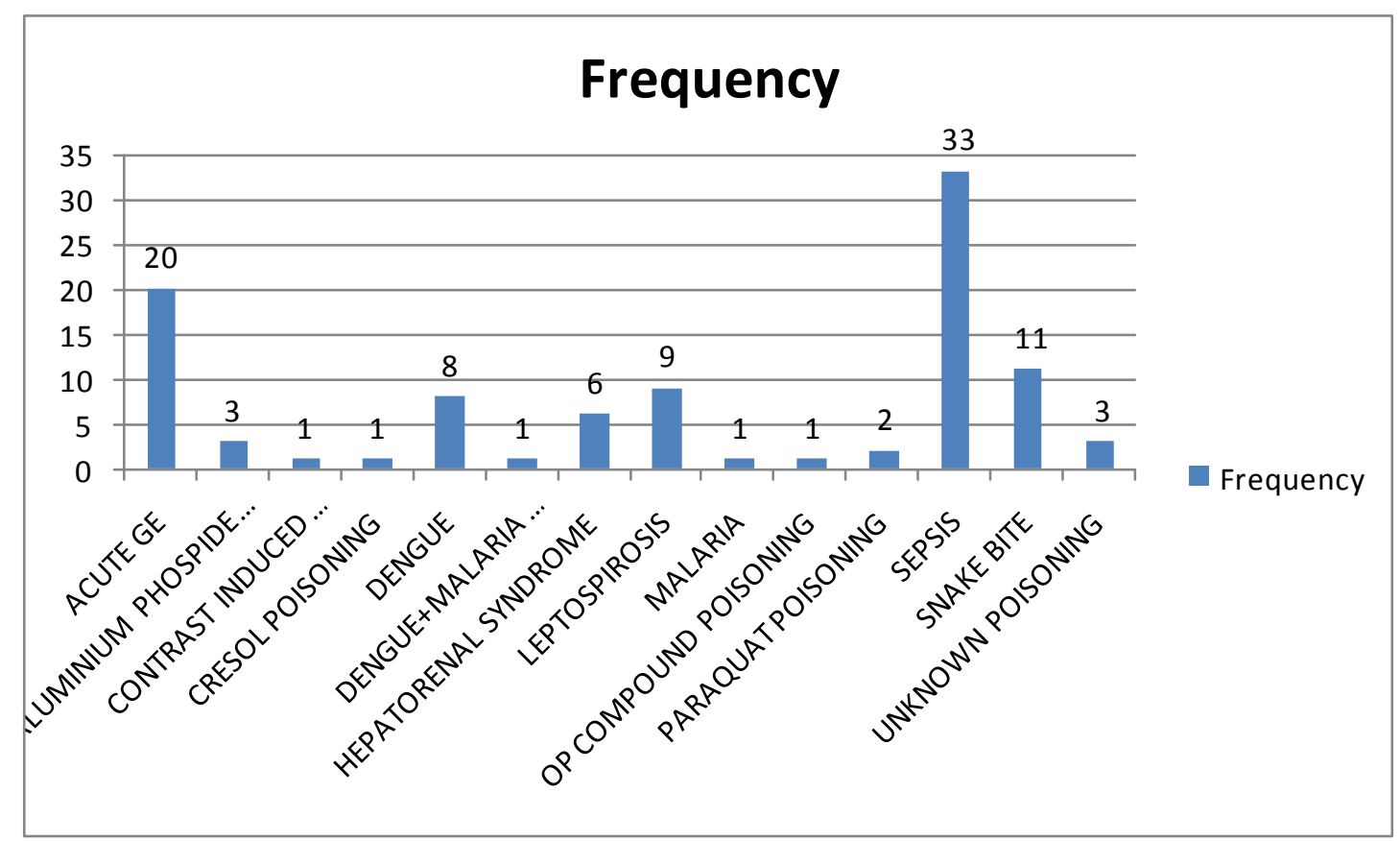

On RIFLE staging mortality in patients with AKI stage failure was $56.2 \%$, injury was $44.4 \%$, and risk is $53.8 \%$ indicating that there was no significant difference in mortality by RIFLE stage. (Table 1)

Table 1. Comparision of Outcome in RIFLE Staging

\begin{tabular}{|l|c|c|}
\hline RIFLE Stage & No Mortality N(\%) & Mortality N(\%) \\
\hline Normal & $14(66.7)$ & $7(33.3)$ \\
\hline Risk & $6(46.2)$ & $7(53.8)$ \\
\hline Injury & $10(55.6)$ & $8(44.4)$ \\
\hline Failure & $21(43.8)$ & $27(56.2)$ \\
\hline
\end{tabular}

On AKIN Staging mortality in Patients with Stage 3 was $56.2 \%$, Stage 2 was $53.8 \%$ and Stage 1 was
$41.2 \%$ indicating that there was no significant difference in mortality by AKIN Stage.(Table 2)

Table 2. Comparision of Outcome in AKIN Staging

\begin{tabular}{|l|c|c|}
\hline AKIN Stage & No Mortality N(\%) & Mortality N(\%) \\
\hline Stage 1 & $20(58.8)$ & $14(41.2)$ \\
\hline Stage 2 & $10(46.2)$ & $8(53.8)$ \\
\hline Stage 3 & $21(43.8)$ & $27(56.2)$ \\
\hline
\end{tabular}

$100 \%$ of patients in normal and risk category with RIFLE were categorized in AKIN stage I.100\% of those in injury were categorized in stage 2 and $100 \%$ of failure categorized in stage 3 AKIN indicating that stage 2 and stage 3 corresponded to injury and failure on RIFLE and those normal on RIFLE corresponded to AKIN Stage 1 Indicating that AKIN increased sensitivity of identifying those with acute kidney injury. (Table 3) 
Table 3. Comparision of RIFLE Vs AKIN Staging

\begin{tabular}{|l|c|c|c|}
\hline RIFLE STAGE & $\begin{array}{c}\text { AKIN Stage 1 } \\
\mathrm{N}(\%)\end{array}$ & $\begin{array}{c}\text { AKIN Stage 2 } \\
\mathrm{N}(\%)\end{array}$ & $\begin{array}{c}\text { AKIN Stage 3 } \\
\mathrm{N}(\%)\end{array}$ \\
\hline Normal & $21(100)$ & 0 & 0 \\
\hline Risk & $13(100)$ & 0 & 0 \\
\hline Injury & 0 & $18(100)$ & 0 \\
\hline Faliure & 0 & 0 & $48(100)$ \\
\hline
\end{tabular}

Table 4.Diagnosis Vs Mortallity

\begin{tabular}{|l|c|c|}
\hline Diagnosis & No Mortality N(\%) & Mortality N(\%) \\
\hline Acute GE & $20(100)$ & 0 \\
\hline Aluminium Phospide poisoning & $2(66.7)$ & $1(33.3)$ \\
\hline Contrast induced nephropathy & $1(100)$ & 0 \\
\hline Cresol Poisoning & $1(100)$ & 0 \\
\hline Dengue & 0 & $8(100$ \\
\hline Dengue+ Malaria & $1(100)$ & 0 \\
\hline Hepatorenal Syndrome & 0 & $6(100)^{*}$ \\
\hline Leptospirosis & $3(33.3)$ & $6(66.7)^{*}$ \\
\hline Malaria & $1(100)$ & 0 \\
\hline Op Compound Poisoning & 0 & $1(100)$ \\
\hline Paraquat Poisoning & $1(50)$ & $1(50)$ \\
\hline Sepsis & $9(27.3)$ & $24(72.7)^{*}$ \\
\hline Snake Bite & $9(81.8)$ & $2(18.2)$ \\
\hline Unknown Poisoning & $3(100)$ & 0 \\
\hline
\end{tabular}

*Significant increase in mortality

In the study 24 patients out of 33 cases of sepsis expired,6 out of 9 patients of leptospirosis expired, 2 out of 11 patients of snake bite expired, 1 out of 3 patients of aluminium phosphide poisoning expired and 1 patients of op compound poisoning expired. All 8 cases of dengue and 6 patients of hepatorenal syndrome expired. All patients of aute GE. Constrast induced nephropathy, cresol poisoning, dengue+malaria coinfection, malaria and unknown poisoning survived.

\section{Discussion}

In this study Patients between the age group18-80 years were analysed and the mean age was $40.22 \pm 14.2$ years and for males it is was $38.4 \pm 12.48$ years and for females it is was $43.5 \pm 16.6$ years out of 100 patients, 65 were male and 35 were female constituting $65 \%$ and $35 \%$ respectively and Male: Female ratio in this study is $1.86: 1$
Lopes et al. in their study of the acute kidney injury in intensive care unit patients: a Comparision Between the RIFLE and the AKIN Classification had patients with mean age of 58.6 years and $59.2 \%$ males. ${ }^{12}$ Chang et al. in their study of acute kidney injury classification: Comparision of AKIN and RIFLE criteria had patients with mean age of 62 years and $70 \%$ males and $30 \%$ females. ${ }^{13}$ Bagshaw SM et al. in their study of comparision of RIFLE and AKIN Criteria for acute kidney injury in critically ill patients had patients with mean age of 61.6 years and $59.5 \%$ males ${ }^{14}$. In the present study mean age was less compared to the other three studies. However percentage of males and females in the present study was similar

\section{Treatment and Mortality}

In this study out of 100 patients, 48 patients required ventilator support which was less compared to Lopes jose et al study in which $84.7 \%$ patients required ventilator support ${ }^{7}$ but 
was similar to Bagshaw et al study in which $52 \%$ patients required ventilator Suoport ${ }^{14}$.

In this study $48 \%$ patients required inotropic support which is comparable to Lopes et al study in which $40 \%$ patient's required inotropic support. $^{12}$

In this study sepsis accounted for 33\% patients which is Comparable to $27.8 \%$ in Bagshaw SM et al study ${ }^{14}, 40.9 \%$ sepsis patients in Lopes Jose et al study and 55\% sepsis Patients in Chang et al study ${ }^{13}$ indicating sepsis as the major cause of
AKI. Sepsis is the leading contributing factor to AKI in Critically ill Patients and generallt portends a worse prognosis.

In this study mean Baseline Blood urea, Serum Creatinine and urine output were similar for both RIFLE and AKIN stages.

In the present study there was $\mathrm{n}$ overall mortality of $49 \%$ which is more than $24.2 \%$ mortality in Bagshaw SM et al study ${ }^{14}$ and lesser than $60.8 \%$ mortality in Chang et al study ${ }^{13}$.

Table 5 : Following is the stage wise comparision between other studies

\begin{tabular}{|l|c|c|c|c|}
\hline \multirow{2}{*}{ STAGE } & \multicolumn{4}{|c|}{ MORTALITY (\%) } \\
\cline { 2 - 5 } & Lopes et al & Chang et al & Bagshaw SM et al & Present Study \\
\hline RIFLE NORMAL & 56.2 & 36.8 & 8.9 & 33.3 \\
\hline RISK & 14.7 & 63.2 & 17.5 & 53.8 \\
\hline INJURY & 11.0 & 69.2 & 27.7 & 44.4 \\
\hline FAILURE & 18.1 & 86.2 & 33.2 & 56.2 \\
\hline AKIN STAGE-1 & 21.1 & 52.6 & 18.5 & 41.2 \\
\hline STAGE-2 & 10.1 & 67.3 & 28.1 & 53.8 \\
\hline STAGE-3 & 19.2 & 84.8 & 32.6 & 56.2 \\
\hline
\end{tabular}

In the present study $13 \%$ (Table 6) patients were classified in RISK category of RIFLE Criteria and $34 \%$ Patients were classified in stage 1 of AKIN Criteria Indicating that AKIN increased sensitivity of identifying those with acute kidney injury(p
Value<0.001).This is Comparable to the conclusion in lopes et al study ${ }^{12}$ and Chang et al Study ${ }^{13}$. However, Bagshaw SM et al study failed to find significant difference between AKIN and RIFLE Criteria ${ }^{14}$.

Table 6: Following is a table depicting the stratification into RIFLE and AKIN criteria in Various Studies.

\begin{tabular}{|l|c|c|c|c|}
\hline CRITERIA & Lopes et al & \multicolumn{3}{|c|}{$\begin{array}{c}\text { PERCENTAGE } \\
\text { Chang et al Bagshaw SM et al present study }\end{array}$} \\
\hline RIFLE & \multicolumn{5}{|c|}{} \\
\hline RISK & 14.7 & 13.1 & 16.2 & 13 \\
\hline INJURY & 11 & 17.9 & 13.6 & 18 \\
\hline FAILURE & 18.1 & 29.9 & 6.3 & 48 \\
\hline AKIN & 21.1 & 19.6 & 18.1 & 34 \\
\hline STAGE 1 & 10.1 & 16.8 & 10.1 & 48 \\
\hline STAGE 2 & 19.2 & 31.6 & 8.9 & \\
\hline STAGE 3 & \multicolumn{5}{|l|}{} \\
\hline
\end{tabular}

\section{Conclusion}

The AKIN criteria improve the sensitivity of the Acute Kidney Injury (AKI) diagnosis over the RIFLE Criteria. The AKIN Criteria do not improve on the ability of the RIFLE criteria in predicting in hospital mortality of critically ill Patients.

\section{Acknowledgement}

We sincerely thank all the individuals who have supported us for the study. We thank Mr Pradeep BK (Biocon) for assistance 


\section{References}

1. Bellomo, R., Ronco, C., Kellum, J. A., Mehta, R. L. \&Palevsky, P. Acute renal failure-definition, outcome measures, animal models, fluid therapy and information technology needs: the Second International Consensus Conference of the Acute Dialysis Quality Initiative (ADQI) Group. Crit Care. 8, R204 (2004).

2. Bellomo, R., Kellum, J. A. \&Ronco, C. Acute kidney injury. Lancet. 380, 756-766 (2012).

3. Chertow, G. M., Burdick, E., Honour, M., Bonventre, J. V. \& Bates, D. W. Acute kidney injury, mortality, length of stay, and costs in hospitalized patients. J Am Soc Nephrol. 16, 3365-3370 (2005).

4. De Mendonça, A. et al. Acute renal failure in the ICU: risk factors and outcome evaluated by the SOFA score. Intensive Care Med. 26, 915-921 (2000).

5. Uchino, S. et al. Acute renal failure in critically ill patients. J Am Med Assoc. 294, 813-818 (2005).

6. Leung, K. C., Tonelli, M. \& James, M. T. Chronic kidney disease following acute kidney injury-risk and outcomes. Nat Rev Nephrol. 9, 77-85 (2012).

7. Susantitaphong, P. et al. World Incidence of AKI: A Meta-Analysis. Clin J Am Soc Nephrol. 8, 1482-1493 (2013).

8. Liangos, $\mathrm{O}$. et al. Epidemiology and outcomes of acute renal failure in hospitalized patients: a national survey. Clin J Am Soc Nephrol. 1, 43-51 (2006).

9. Ricci, Z., Cruz, D. N. \&Ronco, C. Classification and staging of acute kidney injury: beyond the RIFLE and AKIN criteria. Nat Rev Nephrol. 7, 201-208 (2011).

10. Ricci, Z. et al. Practice patterns in the management of acute renal failure in the critically ill patient: an international survey. Nephrol Dial Transpl. 21, 690-696 (2006).
11. Mehta, R. et al. Acute Kidney Injury Network: report of an initiative to improve outcomes in acute kidney injury. Crit care. 11, R31 (2007)

12. Lopes, Paulo Fernandes, Sofia Jorge, Sara Gonçalves, António Alvarez, Zélia Costa e Silva, Carlos França and Mateus Martins Prata Acute kidney injury in intensive care unit patients: a comparison between the RIFLE and the Acute Kidney Injury Network classifications Critical Care 2008 12:R110

13. Chang, Chih-Hsiang; Acute Kidney Injury Classification: Comparison Of Akin And Rifle Criteria Shock: March 2010 - 33( 3): 247-252

14. Sean M. Bagshaw. A comparison of the RIFLE and AKIN criteria for acute kidney injury in critically ill patients Nephrol Dial Transplant (2008) 23: 1569-1574 